\title{
The Accuracy of Contrast-enhanced Computed Tomography Scans to Detect Postpartum Haemorrhage: An Observational Study
}

YUMI MITSUYAMA ( $\sim$ goto8940@gmail.com)

Department of Traumatology and Acute Critical Medicine, Osaka University Graduate School of Medicine, 2-15 Yamadaoka, Suita City, Osaka 565-0871, Japan

\section{YUSUKE KATAYAMA}

Osaka University

KAZUYA OI

Osaka University

JUNYA SHIMAZAKI

Osaka University

KAZUYA MIMURA

Osaka University

MASAYUKI ENDO

Osaka University

TAKESHI SHIMAZU

Osaka University

\section{Research Article}

Keywords: Angiography, Computed tomography, Extravasation, Postpartum hemorrhage

Posted Date: February 2nd, 2021

DOI: https://doi.org/10.21203/rs.3.rs-154428/v1

License: (1) (i) This work is licensed under a Creative Commons Attribution 4.0 International License. Read Full License 


\section{Abstract}

Introduction: Postpartum haemorrhage is a major cause of maternal mortality. Although contrastenhanced computed tomography (CE-CT) is useful to reveal arterial bleeding, its accuracy in postpartum haemorrhage is unclear. The aim of this study was to evaluate the accuracy of CE-CT scanning in detecting postpartum haemorrhage.

Methods: This was a retrospective observational study. We included patients with postpartum haemorrhage treated by emergency physicians in collaboration with obstetricians. We calculated the sensitivity, specificity, and positive and negative predictive values for CE-CT scanning to detect arterial bleeding.

Results: CE-CT scanning was performed in 52 patients, and 31 patients had extravasation. The sensitivity of CE-CT scanning to detect arterial extravasation was $100 \%$ (15/15), specificity was $28.6 \%(2 / 7)$, positive predictive value was $75 \%$ (15/20), and negative predictive value was $100 \%(2 / 2)$.

Conclusion: We showed the sensitivity of CE-CT scanning to detect arterial extravasation in patients with postpartum haemorrhage to be $100 \%$.

\section{Introduction}

Postpartum haemorrhage is a serious obstetric complication and a major cause of maternal mortality that accounts for an estimated 127,000 deaths annually [1]. The main source of bleeding is arterial, but the involvement of venous bleeding in postpartum haemorrhage is not clear [2]. As the haemorrhage progresses, coagulopathy can accelerate bleeding and make it difficult to identify the source [3]. Transvaginal and abdominal echocardiography are used to identify the source of the bleeding, but retroperitoneal haemorrhage is difficult to detect. Moreover, it is very important to identify the source of bleeding to select the appropriate haemostatic treatment.

In the area of trauma, due to its minimal invasiveness and ease of use, contrast-enhanced computed tomography (CE-CT) is used to identify the source of bleeding [4]. Angiography is recommended in the guidelines, especially for arterial bleeding associated with pelvic fractures with stable circulation [5]. The accuracy of CE-CT and angiography has been reported to be high [6]. However, there is little evidence on the use of CE-CT and angiography to identify the source of bleeding in postpartum haemorrhage.

The purpose of this study was to evaluate the accuracy of CE-CT in identifying the site of active bleeding in patients with postpartum haemorrhage.

\section{Methods}

\section{Study design and setting}


This was a retrospective observational study with a study period of 104 months from 1 April 2011 to 31 December 2019. The Trauma and Acute Critical Care Center of Osaka University Hospital, which is located in the northern part of Osaka Prefecture, Japan, annually treats about 1000 severe patients with conditions such as shock, cardiopulmonary arrest, severe trauma, and sepsis. The Center also treats critically ill patients with postpartum haemorrhage in cooperation with obstetricians and gynaecologists. This study included the patients who suffered haemorrhage in the postpartum period and were treated at this institution. On the basis of the diagnosis at hospital admission, we defined the patients with bleeding during the postpartum period as patients with postpartum haemorrhage. Patients experiencing bleeding due to miscarriage were excluded from the study, as were patients with eclampsia and HELLP (haemolysis, elevated liver enzymes, and low platelets) syndrome without postpartum haemorrhage. We also excluded patients who did not undergo CT scanning, were admitted to the ICU, and were not initially treated by emergency physicians. This manuscript was written based on the STROBE statement to assess the reporting of cohort and cross-sectional studies [7]. This study was approved by the institutional review board of Osaka University (approval no. 19509). Consent from individual patients was obtained comprehensively on admission.

\section{Obstetric \& Gynecologic Cooperative System in Osaka Prefecture}

In Japan, there are three levels of perinatal care depending on the risk of pregnancy and delivery [8]. Lowrisk pregnancies and deliveries are managed in small hospitals and clinics, whereas pregnancies and deliveries with moderate risk, such as emergency or abnormal deliveries and general caesarean sections, are managed in regional perinatal medical centres (292 facilities in Japan) that can provide emergency deliveries, caesarean section, and intensive care management for the mothers and new-borns. High-risk pregnancies and deliveries and infants requiring neonatal intensive care are managed at general perinatal medical centres (104 facilities in Japan) that are equipped with maternal-foetal intensive care units and neonatal intensive care units and can provide intensive care for the mothers, foetuses, and new-borns. In Japan, there is a system for referral and transport to higher-level medical facilities according to changes in the risk of pregnancy and delivery throughout the course of the pregnancy. In addition, the Obstetric \& Gynecologic Cooperative System (OGCS) for pregnant women and the Neonatal Mutual Cooperative System for new-borns have been established in Osaka prefecture [9]. When emergency maternal events due to obstetric diseases such as massive bleeding and eclampsia, pulmonary embolism or complications related to obstetric anaesthesia occur, the OGCS allows obstetricians and gynaecologists to directly contact the obstetricians and gynaecologists at the higher-care facility for smooth transport. If serious complications such as placental abruption or severe postpartum haemorrhage occur in small hospitals and clinics, these patients can be transported to higher-level medical facilities through the OGCS for appropriate treatment. Osaka Prefecture has 17 regional perinatal medical centres and 6 general perinatal medical centres, and 34 facilities including 23 of these were registered in the OGCS. Because the most seriously ill pregnant women suffering a traffic accident, cerebral haemorrhage, or cardiopulmonary arrest cannot be adequately treated in normal medical facilities, they are transported to a hospital that has not only an emergency care centre but also a general or regional perinatal medical 
centre (7 facilities in Osaka Prefecture) for intensive care. Our hospital is designated as one of these institutions.

\section{Management of postpartum haemorrhage at Osaka University Hospital}

All critical obstetric patients transported to the Trauma and Acute Critical Care Centre are handled by emergency physicians, obstetricians, and gynaecologists who work together to treat them. The obstetricians and gynaecologists receive the request for patient transfer through the OGCS, and then they notify emergency physicians, anaesthesiologists, and radiologists about the critical obstetric patients being transferred to our hospital. When a woman is transported to our critical care centre, emergency physicians resuscitate her with treatments such as blood transfusion and circulation management. At the same time, the obstetricians and gynaecologists search for the source of bleeding with ultrasonography and inspection of the perineal area and stop postpartum haemorrhage with manual compression of the uterus and balloon compressions.

Our emergency room includes an emergency care room equipped with a computed tomography (CT) table as a treatment bed. Because patients are treated on the CT table, it is possible to perform the entire process from diagnosis to treatment, including resuscitation, without moving them [10]. For CT scanning, a SOMATOM Definition Flash (SIEMENS, Germany) was used. In the protocol for CE-CT scanning for postpartum haemorrhage, we use $95 \mathrm{~mL}$ of iohexol (loberinß) at an infusion rate of $4 \mathrm{~mL} / \mathrm{s}$, and the area from the inferior margin of the sternum to the pelvic floor is examined in two phases of arterial and venous imaging. An Artis Q ceiling-mounted system (Siemens Healthineers ${ }^{\circledR}$ ) was used for angiography.

\section{Endpoints}

The primary endpoint was the presence of arterial bleeding. We defined extravasation on angiography as arterial bleeding in this study. The secondary endpoint was discharge to death.

\section{Statistical analysis}

Continuous variables are indicated by medians (interquartile range [IQR]), and categorical variables are indicated by frequencies and percentages. The shock index (SI), serum lactate level, and blood loss within 24 hours were used as indicators of the severity of the patients on hospital admission. The causes of postpartum haemorrhage were classified into four categories: Tone, Trauma, Tissue and Thrombin [11]. We classified atonic postpartum haemorrhage as Tone; uterine rupture, uterine inversion, and perineal lacerations as Trauma; placenta accreta and placenta previa as Tissue; and disseminated intravascular coagulation and amniotic fluid embolism as Thrombin. We calculated the sensitivity, specificity and positive and negative predictive values of CE-CT scanning to detect arterial bleeding to determine the accuracy of CE-CT scanning to identify the source of arterial bleeding in patients with postpartum haemorrhage. The presence of arterial extravasation on CE-CT scanning was evaluated by two independent radiologists. In addition, we performed subgroup analysis according to the patients' SI. The accuracy of CE-CT scanning to detect arterial bleeding was similarly calculated by dividing the patients 
into two groups: those with SI of less than 1 and those with SI of 1 or more. The Mann-Whitney U test was used to test the continuous variables, and the Fisher exact test was used to test the nominal variables. All analyses were performed using JMP 15 (SAS Institute Inc., Cary, NC, USA).

\section{Results}

Figure 1 shows the flow diagram of this study. Among the 81 patients with postpartum haemorrhage treated by emergency physicians in collaboration with obstetricians and gynaecologists during the study period, CE-CT scanning was performed in 52 patients. Among them, 31 patients showed extravasation on CE-CT scanning, and 21 patients showed no extravasation.

Table 1 shows the patient characteristics divided into four groups according to the presence or absence of extravasation on CE-CT scanning and angiography. The median blood loss was $2900 \mathrm{~g}$ (IQR: 1483$6120 \mathrm{~g}$ ) in the group with extravasation on CE-CT scanning and angiography and $3820 \mathrm{~g}$ (IQR: 2000$6050 \mathrm{~g}$ ) in the group without extravasation on CE-CT scanning and angiography.

In the group with extravasation on CE-CT scanning and angiography, arterial embolization was performed in 19 patients (95.0\%), surgery in 2 patients (10.0\%), and postpartum balloon in 9 patients (45.0\%). In contrast, in the group with extravasation on CE-CT scanning and without angiography, surgery was performed in 5 patients (45.5\%), and postpartum balloon in 8 patients (72.7\%). In the group without extravasation on CE-CT and with angiography, embolization was performed in one patient. In the group without extravasation on CE-CT and angiography, surgery was performed in 3 patients (14.3\%) and postpartum balloon in 7 patients (36.8\%). The group receiving the highest dose of RBC suspension was the group with extravasation on CE-CT and angiography, at $1680 \mathrm{~g}$ (IQR: 560-3640 g).

Table 2 shows the relationship between the presence of extravasation on CE-CT scanning and arterial bleeding. The sensitivity of detecting arterial extravasation on CE-CT scanning was 100\% (15/15), the specificity was $28.6 \%$ (2/7), the positive predictive value was $75 \%(15 / 20)$, and the negative predictive value was $100 \%(2 / 2)$.

Table 3 shows the results of the subgroup analysis of patients with SI less than 1. Of these, 18 patients underwent angiography. Twelve patients had extravasation on CE-CT scanning and 1 patient did not. The sensitivity of extravasation on CE-CT scanning for arterial bleeding was $100 \%$ (12/12), the specificity was $16.7 \%(1 / 6)$, the positive predictive value was $70.6 \%(12 / 17)$, and the negative predictive value was $100 \%$ $(1 / 1)$.

Table 4 shows the results of the subgroup analysis of patients with SI of 1 or more. Of these patients, 4 underwent angiography. The sensitivity of extravasation on CE-CT for arterial bleeding was 100\% (3/3), the specificity was $100 \%(1 / 1)$, the positive predictive value was $100 \%(3 / 3)$, and the negative predictive value was $100 \%(1 / 1)$.

All 52 patients with postpartum haemorrhage survived and were discharged from the hospital. 


\section{Discussion}

In the present study, we showed that in the patients with postpartum haemorrhage, the sensitivity of detecting arterial extravasation on CE-CT scanning was $100 \%$ and the negative predictive value was also $100 \%$. It is important to identify the source of bleeding when planning a haemostatic strategy. In the case of arterial bleeding, there are options other than surgery as a haemostatic strategy, such as uterine artery embolization. Therefore, this study, which showed for the first time, to our knowledge, the accuracy of CECT scanning in postpartum haemorrhage, may be useful in planning strategies to treat postpartum haemorrhage.

To our best knowledge, there are no reports of the accuracy of detecting arterial extravasation on CE-CT scanning in postpartum patients, but there are many reports of its utility in severe trauma patients. Salimi et al. reported that the sensitivity of CE-CT scanning for intra-abdominal organ injury was $100 \%$ for liver injury and $86.6 \%$ for splenic injury [12]. Dreizin et al. reported that the sensitivity and specificity of CE-CT scanning to search for arterial bleeding in pelvic trauma were $80 \%$ and $93 \%$, respectively [13]. Because the presence of arterial injury can be identified by extravasation on CE-CT scanning, CE-CT scanning can rapidly identify the site of injury in parenchymal organ injury and pelvic trauma [14]. Thus, the identification of arterial extravasation with CE-CT scanning in trauma is a useful diagnostic tool due to its high sensitivity and negative predictive value [15]. In the present study, the identification of arterial extravasation on CE-CT scanning was highly sensitive in postpartum haemorrhage, and its presence on CE-CT imaging may be useful as a screening test to detect arterial bleeding in postpartum patients.

The presence of arterial bleeding is important in planning a treatment strategy because treatment options such as trans-catheter arterial embolization (TAE) are available in addition to surgical treatment. According to the Eastern Association for Surgery of Trauma guidelines, when the cause of bleeding is intra-abdominal haemorrhage or intra-abdominal organ damage, TAE is a treatment option as long as the patient's vital signs are stable [4]. Furthermore, when the cause of bleeding is retroperitoneal haemorrhage such as from pelvic fracture, TAE is the first choice for bleeding control, even if the patient's vital signs are unstable [16]. Kuo et al. reported that in trauma with haemorrhagic shock, there were cases requiring TAE among those who did not initially show extravasation on CE-CT scanning. They reported that in patients with pelvic fractures, $19.5 \%$ (30/154) of those without extravasation on CE-CT scanning underwent TAE, and these patients were accompanied by hypotension (extravasation $(+)$ vs. extravasation (-); $68 \mathrm{mmHg}$ vs. $129 \mathrm{mmHg}$ [median]) on hospital arrival [17]. However, the effectiveness of uterine artery embolization for postpartum haemorrhage has also been noted in several studies [18], but the rapid identification of the source of bleeding is essential in the case of critical postpartum haemorrhage. Intraperitoneal and retroperitoneal haemorrhage can occur in postpartum haemorrhage, and in the present study, the sensitivity of detecting arterial extravasation with CE-CT scanning in postpartum haemorrhage was $100 \%$ not only in the patients with shock but also in those without shock.

Surgery is the first choice for haemostatic treatment in critical trauma patients whose vital signs are unstable. Resuscitative endovascular balloon occlusion of the aorta (REBOA) is a technique used to 
control subdiaphragmatic bleeding. REBOA is used as a damage control strategy for trunk trauma with haemorrhagic shock and also preoperatively and intraoperatively for surgery and transcatheter therapy. The efficacy of REBOA has been reported for postpartum haemorrhage with unstable circulation $[19,20]$. In the present study, blood loss was highest in the group with extravasation on CE-CT and without angiography, (3820 g, IQR; 2000-6050 g), and 4 patients required hysterectomy for haemostatic treatment. Although the median SI in this group was 1.0, the condition of the patients required urgent haemostatic treatment via surgery. Hysterectomy cannot preserve fertility, but if REBOA can be used as a bridging therapy until angiography, it may be possible to preserve the uterus.

\section{Limitations}

There are several limitations in this study. First, this was a single-centre retrospective study, so its general validity may be low. The accuracy of CE-CT scanning should be validated by collaborating with other institutions in the future. Second, because we defined extravasation on angiography as arterial bleeding in this study, it was unknown whether the patients who showed no extravasation on CE-CT scanning and angiography actually had arterial bleeding. Finally, since this study is an observational study, there may be some unknown confounding factors.

\section{Conclusion}

The present study revealed that the sensitivity of detecting arterial extravasation on CE-CT scanning in postpartum haemorrhage was $100 \%$ and the negative predictive value was also $100 \%$.

\section{Abbreviations}

CT

computed tomography; CE-CT:contrast-enhanced computed tomography; OGCS:Obstetric \& Gynecologic Cooperative System; SI:shock index; TAE:trans-catheter arterial embolization; REBOA:resuscitative endovascular balloon occlusion of the aorta; IQR:interquartile range

\section{Declarations}

\section{Ethics approval and consent to participate}

This study followed the principles of the Declaration of Helsinki.

This study was approved by the institutional review board of Osaka University (approval no. 19509). Informed consent was obtained from all study participants.

\section{Consent for publication}

Not applicable. 
Availability of data and materials

The datasets used during the current study are available from the corresponding author on reasonable request.

\section{Competing interests}

None.

\section{Funding}

This research did not receive any specific grant from funding agencies in the public, commercial, or notfor-profit sectors.

\section{Authors' contributions}

YM was responsible for formulating the overall research question, designed the study and wrote the manuscript. YK helped to provide statistical advice on the design of the study, carry out the study and write the draft. KO collected and analyzed the data. JS interpreted the data and provided important input to the manuscript. KM and ME took part in the study concept and critical revision of the manuscript. TS organized and supervised the conduction of the study. All authors read and approved the final manuscript.

\section{Acknowledgements}

The authors thank all of the obstetricians, gynaecologists, emergency medical service personnel, nurses and emergency physicians and the patients who contributed to this study.

\section{References}

1. World Health Organization (2008) Reducing the Burden: Postpartum Hemorrhage.

2. Chen C, Lee SM, Kim JW, Shin JH. Recent update of embolization of postpartum hemorrhage. Korean J Radiol 2018;19(4):585-96.

3. Collins P, Abdul-Kadir R, Thachil J, Subcommittees on Women's Health Issues in Thrombosis and Haemostasis and on Disseminated Intravascular Coagulation. Management of coagulopathy associated with postpartum hemorrhage: guidance from the SSC of the ISTH. J Thromb Haemost 2016;14(1):205-10.

4. Stassen NA, Bhullar I, Cheng JD, Crandall M, Friese R, Guillamondegui O, et al. Nonoperative management of blunt hepatic injury: an Eastern Association for the Surgery of Trauma practice management guideline. J Trauma Acute Care Surg 2012;73(5 Suppl 4):S288-93.

5. Cullinane DC, Schiller HJ, Zielinski MD, Bilaniuk JW, Collier BR, Como J, et al. Eastern Association for the Surgery of Trauma practice management guidelines for hemorrhage in pelvic fracture-update 
and systematic review. J Trauma 2011;71(6):1850-68.

6. Maturen KE, Adusumilli S, Blane CE, Arbabi S, Williams DM, Fitzgerald JT, et al. Contrast-enhanced CT accurately detects hemorrhage in torso trauma: direct comparison with angiography. J Trauma 2007;62(3):740-5.

7. von Elm E, Altman DG, Egger M, Pocock SJ, Gøtzsche PC, Vandenbroucke JP; STROBE Initiative. The Strengthening the Reporting of Observational Studies in Epidemiology (STROBE) statement: guidelines for reporting observational studies. J Clin Epidemiol 2008;61(4):344-9.

8. Nishida H. Perinatal health care in Japan. J Perinatol 1997;17(1):70-4.

9. Kanagawa T, Yoshimatsu J, Mitsuda N; Obstetrics and Gynecology Mutual Corporative System (OGCS). Need for annual survey of severe maternal morbidity: The Osaka action agenda. J Obstet Gynaecol Res 2016;42(7):906.

10. Kinoshita T, Yamakawa K, Matsuda H, Yoshikawa Y, Wada D, Hamasaki T, et al. The survival benefit of a novel trauma workflow that includes immediate whole-body computed tomography, surgery, and interventional radiology, all in one trauma resuscitation room: a retrospective historical control study. Ann Surg 2019;269(2):370-6.

11. American College of Obstetricians and Gynecologists. ACOG Practice Bulletin: Clinical Management Guidelines for Obstetrician-Gynecologists Number 76, October 2006: postpartum hemorrhage. Obstet Gynecol 2006;108(4):1039-47.

12. Salimi J, Bakhtavar K, Solimani M, Khashayar P, Meysamie AP, Zargar M. Diagnostic accuracy of CT scan in abdominal blunt trauma. Chin J Traumatol 2009;12(2):67-70.

13. Dreizin D, Liang Y, Dent J, Akhter N, Mascarenhas D, Scalea TM, et al. Diagnostic value of CT contrast extravasation for major arterial injury after pelvic fracture: a meta-analysis. Am J Emerg Med 2020;38(11):2335-42.

14. Willmann JK, Roos JE, Platz A, Pfammatter T, Hilfiker PR, Marincek B, et al. Multidetector CT: detection of active hemorrhage in patients with blunt abdominal trauma. AJR Am J Roentgenol 2002;179(2):437-44.

15. Peitzman AB, Makaroun MS, Slasky BS, Ritter P. Prospective study of computed tomography in initial management of blunt abdominal trauma. J Trauma 1986;26(7):585-92.

16. Awwad A, Dhillon PS, Ramjas G, Habib SB, Al-Obaydi W. Trans-arterial embolisation (TAE) in haemorrhagic pelvic injury: review of management and mid-term outcome of a major trauma center. CVIR Endovasc 2018;1(1):32.

17. Kuo LW, Yang SJ, Fu CY, Liao CH, Wang SY, Wu SC. Relative hypotension increases the probability of the need for angioembolisation in pelvic fracture patients without contrast extravasation on computed tomography scan. Injury 2016;47(1):37-42.

18. Ganguli S, Stecker MS, Pyne D, Baum RA, Fan CM. Uterine artery embolization in the treatment of postpartum uterine hemorrhage. J Vasc Interv Radiol 2011;22(2):169-76.

19. Stensaeth KH, Sovik E, Haig IN, Skomedal E, Jorgensen A. Fluoroscopy-free Resuscitative Endovascular Balloon Occlusion of the Aorta (REBOA) for controlling life threatening postpartum 
hemorrhage. PLoS One 2017;12(3):e0174520.

20. Brenner M, Teeter W, Hoehn M, Pasley J, Hu P, Yang S, et al. Use of resuscitative endovascular balloon occlusion of the aorta for proximal aortic control in patients with severe hemorrhage and arrest. JAMA Surg 2018;153(2):130-5.

\section{Tables}

Due to technical limitations, table 1-4 is only available as a download in the Supplemental Files section.

\section{Figures}

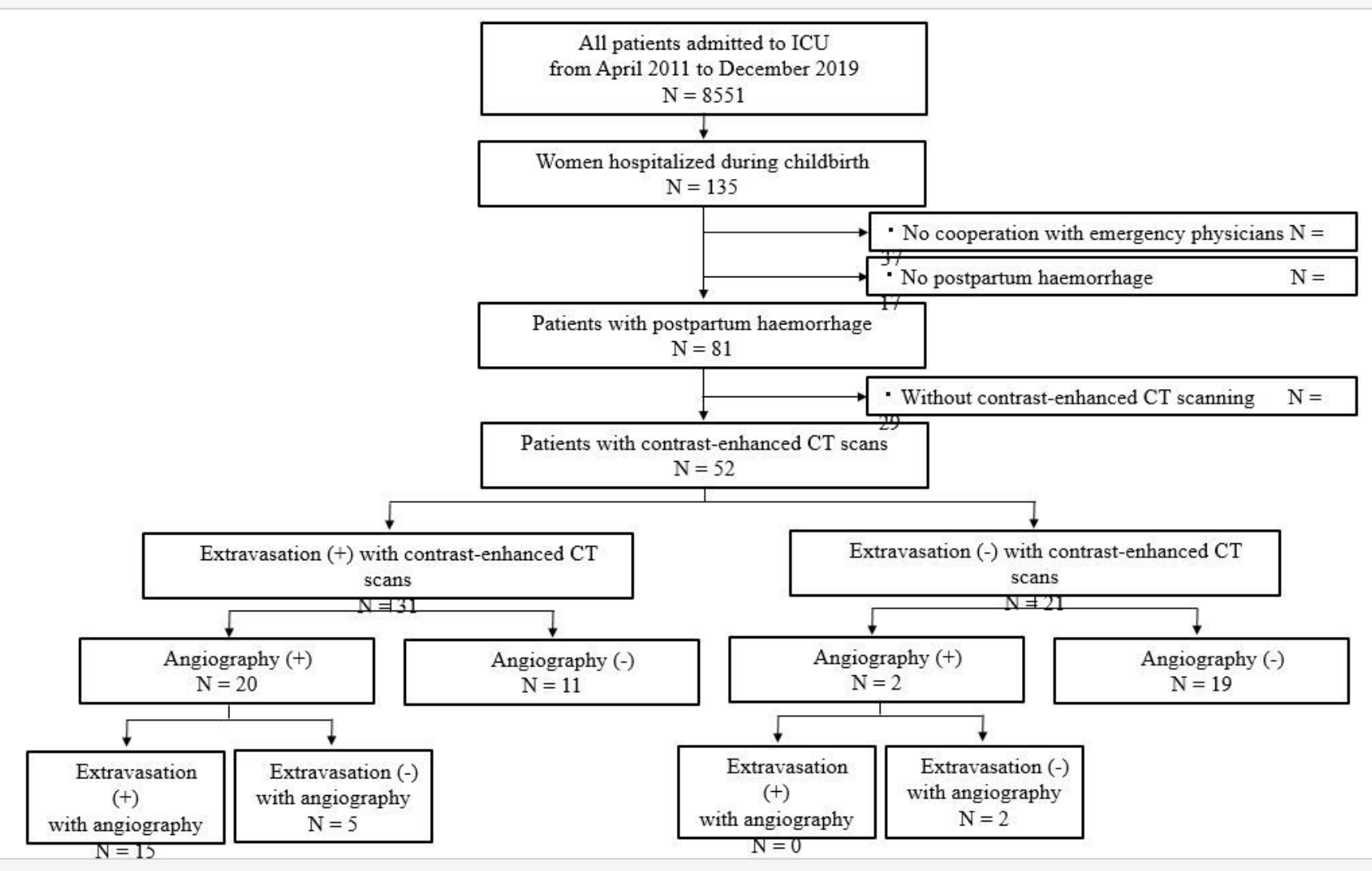

Figure 1

Patient flow in the study. ICU, intensive care unit; CT, computed tomography

\section{Supplementary Files}

This is a list of supplementary files associated with this preprint. Click to download.

- Tables.docx 